\title{
ADAPTIVE OPTICS NIR IMAGING OF R136 IN 30 DORADUS: THE STELLAR POPULATION OF A NEARBY STARBURST
}

\author{
B.BRANDL, B.J.SAMS, F.BERTOLDI, A.ECKART, R.GENZEL, \\ S.DRAPATZ, R.HOFMANN, M.LÖWE AND A.QUIRRENBACH \\ Max-Planck-Institut für Extraterrestrische Physik \\ Giessenbachstrasse, 85740 Garching, Germany
}

\section{Introduction}

The center of the stellar association in 30 Doradus, R136 (HD 38268), is an unusually high concentration of massive and bright $\mathrm{O}, \mathrm{B}$ and Wolf-Rayet stars and represents the closest example of an intense starburst region.

Our analysis investigates this region via high angular resolution NIR adaptive optics observations. We used ESO's adaptive optics sytem COME ON+ (Beuzit et al. 1995) at the 3.6m telescope at La Silla, Chile, together with MPE's NIR camera SHARP II (Brandl 1995a).

For the first time at NIR wavelengths we were able to resolve the crowded core of R136a into its individual components. The faintest sources detected are 20th magnitude in K (Brandl 1995b).

Our maps have spatial resolution comparable to that of recent WFPC2 images (Hunter et al. 1995), allowing us to combine our $\mathrm{H}$ and $\mathrm{K}$-band maps with HST's U, V and I filter bands. We fit the multicolor stellar luminosities over $\sim 600$ stars to a large grid of model magnitudes. This fit approach enables us to derive age, initial mass, and foreground extinction for each star individually. A comprehensive description of this analysis is given in Brandl et al. (1996).

\section{Results}

We detected and subclassified Wolf-Rayet WN-type stars spectroscopically, according to their He II $(2.189 \mu \mathrm{m})$ and $\operatorname{Br} \gamma(2.166 \mu \mathrm{m})$ line emission. We did not detect red supergiants in our field of view but we found 100 faint red sources, which are most likely low or intermediate mass pre-main-sequence 
stars. The stellar ages and ZAMS masses of $\sim 600$ extinction corrected mainsequence $\mathrm{O}, \mathrm{B} \& \mathrm{~A}$ stars permit accurate mass-based dynamical calculations.

We constrain the cluster age between $3 \mathrm{Myr}$ and $7 \mathrm{Myr}$ from the presence of WR stars and the lack of red supergiants. The derived WR to $\mathrm{O}$ star ratio of $4 \%$ could be explained with a cluster age of $3.7 \pm 0.5 \mathrm{Myr}$ (Parker et al. 1995).

Within our 12 !" $8 \times 12$ !" 8 field of view the mass function has the slope $\Gamma=-1.59 \pm 0.10$. The mass function steepens with radial distance from $\Gamma=-1.33 \pm 0.16$ at the cluster center to $\Gamma=-1.63 \pm 0.18$ at $1 \mathrm{pc}$.

We fit the projected mass density distribution to a King (1962) model. Taking all detected stars $\left(M \gtrsim 4 \mathrm{M}_{\odot}\right)$ into account, we find $r_{c}=0 . " 97 \pm 0 . " 07$ $(0.24 \mathrm{pc})$. If only the more massive stars $\left(M \gtrsim 25 \mathrm{M}_{\odot}\right)$ are considered, the derived core radius decreases down to 0.2 (figure 1 ). Hence the "core" of massive stars is smaller than that of light stars. Calculating the half-mass relaxation time of the O-star population we find $t_{r h(O)} \approx 10^{6} \mathrm{yr}$, which is several times smaller than the derived cluster age. Dynamical relaxation processes therefore certainly play an important role in producing the observed radial variations of the present day mass function.
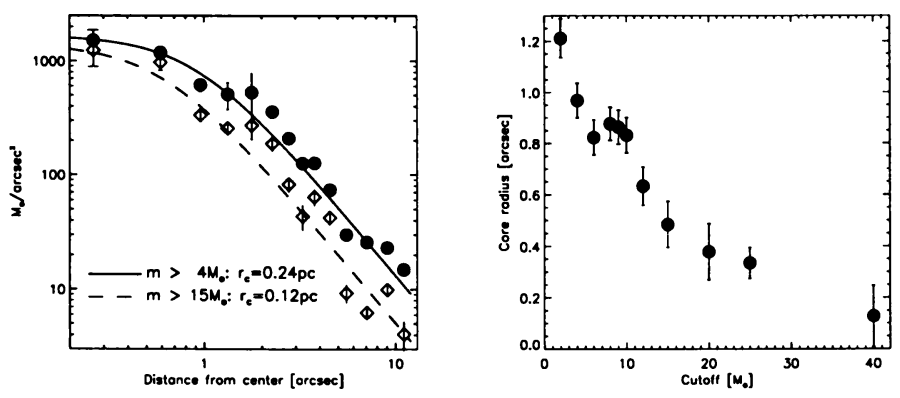

Figure 1. Left: Fit to the stellar mass density distribution, excluding the first data point. Right: The core radius as a function of the detected mass range.

\section{References}

Beuzit, J.-L. et al. 1995, A\&A, in preparation.

Brandl, B. 1995a, Ph.D.thesis, Ludwig-Maximilians-Universität München.

Brandl, B. 1995b, S\&T, August 1995, 14.

Brandl, B., Sams, B.J., Bertoldi, F., Eckart, A., Genzel, R., Drapatz, S., Hofmann, R., Loewe, M., Quirrenbach, A. 1996, submitted to ApJ.

Hunter, D. A., Shaya, E. J., Holtzman, J. A., Light, R. M., O'Neil Jr., E. J., Lynds, R. 1995, ApJ 448, 179.

King, I.R. 1962, AJ 67, 471.

Parker, J. W., Heap, S. R., Malumuth, E. M., 1995, ApJ 448, 705. 